\title{
Application of preoperative computed tomographic lymphography for precise sentinel lymph node biopsy in breast cancer patients
}

Shishuai Wen ${ }^{1,2,3 \dagger}$, Yiran Liang ${ }^{1 \dagger}$, Xiaoli Kong ${ }^{1}$, Baofeng Liư ${ }^{4}$, Tingting Ma' ${ }^{1}$, Yeqing Zhou', Liyu Jiang ${ }^{1}$, Xiaoyan Li $\mathrm{i}^{1}$ and Qifeng Yang ${ }^{1,5,6^{*}}$

\begin{abstract}
Background: In light of the extensive application of sentinel lymph node biopsy (SLNB) in clinically node-negative breast cancer patients and the recently investigated failure of SLNB after lumpectomy, it has become important to explore methods for preoperative mapping of sentinel lymph nodes (SLNs) and their lymphatics to direct precise SLNB and improve the identification rate of SLNs.
\end{abstract}

Methods: Twenty-seven patients with suspected breast cancer based on the results of the clinical examination and imaging were enrolled in the study. Computed tomographic lymphography (CTLG) followed by CT three-dimensional reconstruction was performed to determine the localization of SLNs and lymphatics on the body surface preoperatively. Intraoperatively combined staining with methylene blue and indocyanine green was used to evaluate the accuracy and feasibility of CTLG.

Results: SLNs and lymphatics from the breast were identified using CTLG in all patients, and preoperative SLNs and lymphatics localization on the body surface showed a significant role in the selection of operative incision and injection points. The accuracy rate of SLN and lymphatic detection by CTLG was $92.6 \%$ compared with intraoperatively combined staining. Moreover, preoperative CTLG performed well in SLN number detection, and the accuracy rate was $95.2 \%$.

Conclusion: We evaluate the procedure and application of preoperative CTLG in the superficial localization of SLNs and lymphatics, which may lead to a decreased incidence of cutting off the lymphatics of SLNs and consequently more rapid and accurate SLN detection. This method promotes personalized SLN mapping, providing detailed information about the number and anatomical location of SLNs and lymphatics for adequate surgical planning for breast cancer patients.

Keywords: Sentinel lymph node biopsy, Preoperative SLNs and lymphatics localization, Computed tomographic lymphography, CT three-dimensional reconstruction

*Correspondence: qifengy_sdu@163.com

'Shishuai Wen and Yiran Liang contributed equally to this work.

1 Department of Breast Surgery, General Surgery, Qilu Hospital

of Shandong University, Wenhua Xi Road No. 107, Jinan 250012,

Shandong, China

Full list of author information is available at the end of the article

\section{Background}

Breast cancer is the predominant malignancy among women worldwide and accounts for approximately $15 \%$ of cancer-related deaths in women [1]. The axillary lymph node (LN) status is essential for the prognosis and treatment of breast cancer patients. Indeed, the 5-year overall survival (OS) rate for breast cancer patients with 
LN metastasis is reduced by $40 \%$ compared to that of patients with negative lymph nodes [2]. Therefore, it is important to determine the lymph node status accurately. Mastectomy and axillary lymph node dissection (ALND) have been widely accepted as the gold standard treatment for patients with breast cancer for decades. Although ALND is a reliable procedure to identify nodal metastasis and maintain locoregional disease control, it leads to notable postoperative complications, including pain, numbness, loss of strength, loss of sensitivity, and edema [3, 4], which significantly affect the quality of life of patients.

Sentinel lymph nodes (SLNs) refer to the initial lymph nodes to receive lymphatic drainage from the primary tumor. SLN is reported to be a predictive factor of metastatic spread to the respective regional nodal basins, and sentinel lymph node biopsy (SLNB) has gradually substituted ALND as the standard surgical procedure for clinically node-negative patients with early-stage breast cancer due to significantly fewer complications and indiscrimination between ALND and SLNB in survival and locoregional disease control [5-11]. With the development of dyes and tracers, various intraoperative detection methods of SLNs have been revealed, including methylene blue dye, indocyanine green fluorescence and radioactive colloid. The combination of radioactive colloid and blue dye, which exhibits a higher SLN detection rate $(>90 \%)$ and a lower false negative rate $(<5-10 \%)$, is the internationally recommended standard tracer method for SLNB [12]. However, given the risk of exposure to radiation, training of doctors, legislative requirements, the need for professional equipment, and the cost, the application of radioactive colloids in China is limited [13]. On the other hand, the dye method is simple and easy to master after training and is the widely used tracing method for SLNB in China.

For preoperative biopsy, fine needle aspiration cytology (FNAC) and core needle biopsy (CNB) are widely recommended for breast lesion diagnosis [14]. However, previous studies revealed that percutaneous biopsies may lead to spreading of malignant breast cells following the needle tract or hematogenous spillage $[15,16]$, whereas other studies reported that displaced malignant cells might not be viable due to the immune system $[17,18]$. Although several case studies reported the recurrence of breast cancer in the needle tract [19], cohort studies have not found any association between local recurrence or overall survival in breast cancer patients [20, 21]. However, there seems to be a prevalent belief among patients that percutaneous biopsies might promote the spread of cancer [22]. In addition, there exists a false negative rate for FNAC and CNB due to the experience of surgeons and pathologists and the small volume of breast lumps
$[23,24]$. Moreover, the waiting time for paraffin pathology diagnosis after biopsies is too long, which restricts its application to some extent. Therefore, excision biopsy might be performed when other percutaneous biopsies are not feasible. When excision biopsy was performed for the intraoperative pathological assessment before SLNB, the lymphatic drainage of SLNs might be cut off, potentially leading to identification failure of SLNs. Moreover, lymph flow rerouting and fatty axilla could also reduce the accumulation of radiocolloids and blue dye in SLNs [25]. Therefore, preoperative mapping of SLNs and their lymph vessels (LVs) is helpful for avoiding inaccurate dissection and consequently improving the identification rate of SLNB.

Recently, computed tomographic lymphography (CTLG), a safe technique allowing preoperative SLN navigation, has been proposed for SLN mapping in various cancers [26-28]. These high-resolution images could be used for three-dimensional reconstruction and produce favorable results for providing precise images of the SLNs and their afferent LVs with the surrounding anatomy [25]. Moreover, CTLG could be performed during routine CT scans to preoperatively screen distant metastasis for breast cancer patients, adding little costs or time to the procedure [29]. In this study, we performed CTLG to preoperatively localize SLNs and lymph vessels in patients with early breast cancer and evaluated the usefulness and accuracy of CTLG for the localization of SLNs.

\section{Methods \\ Patients}

Between June 2017 and November 2017, 27 patients with suspected breast cancer based on the results of clinical examination and imaging methods were included in this study. The median age of these patients was 51 years (range 30-65 years). In 27 patients, the lymph nodes were clinically negative. The axillary lymph nodes of patients were negative as determined through clinical physical examination and imaging examination (mammography or ultrasound). No distant metastasis of these patients was detected. Patients with pregnancy, thyroid disease, contraindication to $\mathrm{CT}$ or allergy to the contrast agent were excluded. The detailed patient characteristics are presented in Additional file 1: Table S1. Informed consents were obtained from the patients before the tests. The study was approved by the Ethical Committee of Qilu Hospital of Shandong University (KYLL-2016-231).

\section{Computed tomographic lymphography (CTLG)}

CTLG is a safe technique allowing SLN navigation with satisfying results, which had been proved in several cancers. Radiation exposure to patients is negligible in SLNB with CTLG, about $0.1 \mathrm{mSv}$. Patients were placed in the 
supine position with both arms elevated above the head and the elbows flexed. The contrast solution was composed of $10 \mathrm{ml}$ iopamidol 370 (Shanghai Bracco sine pharmaceutical crop, Shanghai, China), $5 \mathrm{ml}$ lidocaine hydrochloride injection, and $2.5 \mathrm{ml}$ normal saline. After subcutaneous injection of $8-10 \mathrm{ml}$ contrast solution in the areola and 2-3 $\mathrm{ml}$ contrast solution in the peritumoral area, the injection regions were messaged for $30 \mathrm{~s}$. Then, the breast and ipsilateral axillary region with were covered with radiopaque grid (N. TLMT crop, Nan Jing, China) (Fig. 1a). CT was performed with a multisection scanner (SOMATOM Force 75,585, Siemens, German) by using axial scanning $(120 \mathrm{kVp} ; 100 \mathrm{~mA}$; slice thickness, $1.0 \mathrm{~mm}$; slice interval, $0.5 \mathrm{~mm}$; field of view, 512 ; speed, 0.75) [30]. After adjustment of color contrast and view angle, virtual 3D lymphography was obtained using the three-dimensional reconstruction software of the CT scanner or SPECTRA system, which could directly

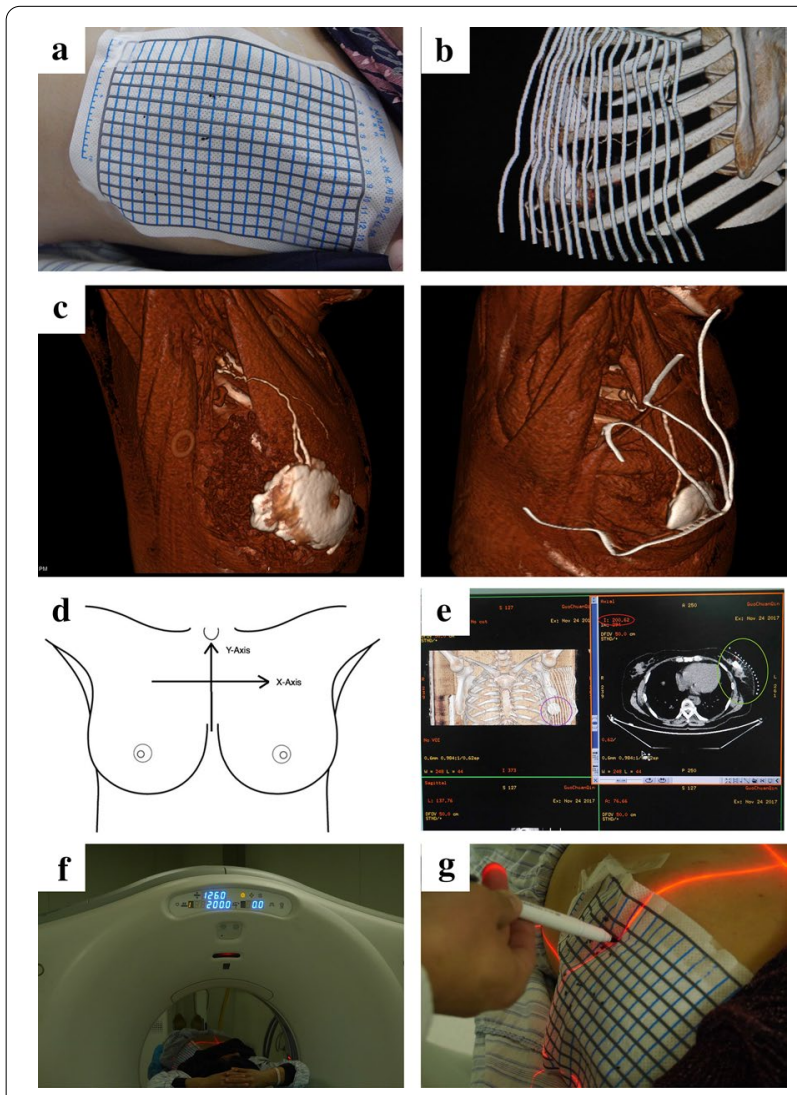

Fig. 1 The procedure of preoperative CTLG. a Fix the radiopaque grid in breast and ipsilateral axilla. b Show the lymphatic drainage pathway using three-dimensional reconstruction of the CT scanner. c Representative images of three-dimensional reconstruction using SPECTRA. $\mathbf{d}$ Virtual $X$ - and $Y$ - axis on the human body. e Operation interface of $C T$ three-dimensional reconstruction software and CT scanner. f Adjust the bed position to "index 200.0". g Localize the specific points according to $X$ - and $Y$ - coordinates display the location of SLNs, lymphatics, and parallel lead strings attached to breast skin (Fig. 1b-c).

\section{Preoperative superficial localization of SLNs and lymphatics}

The human body was placed in a virtual $\mathrm{X}-\mathrm{Y}$ coordinate system (Fig. 1d). The lymph node or crucial point of the lymphatics is chosen in the 3D lymphography image (the red cross in purple circle, Fig. 1e) that corresponds to a point in the CT operation interface at the same location (the red cross in green circle). The index (' $\mathrm{I}=200.62^{\prime}$ in red circle), representing the sagittal position of the CT bed, is the Y-coordinate. The X-coordinate of the red cross is determined by the location relative to the parallel lead strings (the middle position of the second and third strings from medial to lateral). The X-and Y-coordinates of several crucial points, including the starting points, ending points (SLN), and inflexion points, were recorded in the same manner. Adjust the position of the CT bed based on the Y- coordinate (Fig. 1f); the red laser line cast on the human body indicates the Y-axis. The location of a specific point is at the joint point of the red laser line cast on the human body and the X-coordinate based on the radiopaque grid (Fig. 1g). The positions of all points on the body surface were marked and connected to obtain an integral lymphatic drainage pathway.

\section{Methylene blue and indocyanine green staining}

Briefly, $0.6 \mathrm{ml}$ methylene blue was injected subcutaneously, and indocyanine green was injected subcutaneously at the same point after $3 \mathrm{~min}$. A near-infrared camera (Ming De, China) was used to detect fluorescent lymphatics and SLNs. SLNs were also detected following blue staining and fluorescent lymph vessels. The visualization of SLNs and lymphatics was categorized into 3 degrees, as shown in Additional file 1: Table S2. $\mathrm{SLN}++$ or $\mathrm{SLN}+$ was defined as successful visualization of the SLN, and LV++or LV+ was defined as successful visualization of the LV. Successful visualization of both SLNs and LVs was defined as successful lymphography. The accuracy of CTLG was evaluated by comparing the consistency between preoperative CTLG and intraoperative detection.

\section{Result}

\section{Preoperative localization of SLNs and lymphatics using} CTLG

The SLNs and their LVs were successfully located in all 27 patients using preoperative CTLG with a $100 \%$ successful identification rate. Both SLNs and LVs were completely visualized (SLN++/LV++) in 19 patients. In four patients, part of the SLNs visualized, and 3 of these patients had significantly enlarged lymph nodes. There 
Table 1 Number of patients in different categories of CTLG

\begin{tabular}{llllc}
\hline & SLN ++ & SLN+ & SLN- & Overall N \\
\hline LV++ & 19 & 3 & 0 & 22 \\
LV+ & 4 & 1 & 0 & 5 \\
LV- & 0 & 0 & 0 & 0 \\
$N$ & 23 & 4 & 0 & 27 \\
\hline
\end{tabular}

SLN, sentinel lymph node; LV, lymph vessel

were 5 patients with only part of the LV being visualized. Of those 5 patients, the initial part was invisible in 3 patients, whereas intermediate and terminal parts were invisible in 1 patient, respectively. Both the SLN and LV were simultaneously partially visible in 1 patient. The detailed number of patients in different categories of CTLG is shown in Table 1. Certain rules exist about the courses of LVs and the connection patterns between SLNs and LVs. In the research, one LV connecting with one SLN was the most frequent pattern followed by the pattern in which one LV connects with two SLNs. Patterns in which multiple LVs connect with single or multiple SLNs were also observed; but these events were rare. The detailed connection patterns are shown in Additional file 1: Table S3. Although iopamidol was injected in both the areola and skin over the tumor, all of the observed LVs were stretched from the subareolar lymphatic plexus, flowing into SLNs, and no LV was stretched from the surface of the tumor. Detailed information about the starting points of lymphatics is presented in Additional file 1: Table S4.

\section{The consistency between preoperative SLN and lymphatics localization and intraoperative detection}

The localization of LVs and SLNs was further verified by combined staining with methylene blue and indocyanine green fluorescence during operation. Using the result of intraoperative detection as a reference, the consistency indicates that preoperative localization revealed exactly the same course of lymphatics or completely the same trunk with few variable small branches. Moreover, the detection of more or less small branches of lymphatics, which did not connect with any SLNs, did not affect the consistency. However, different detection of the course or number of trunks by preoperative localization was considered inconsistent with intraoperative detection. A typical case that indicated the consistency between the two methods is presented in Fig. 2.

Of the 27 patients who were successfully localized through preoperative CTLG, 25 patients had consistent SLNs and LVs. The accuracy rate of SLN and lymphatic detection of preoperative CTLG was 92.6\% (25/27 cases) compared with combined staining with methylene blue

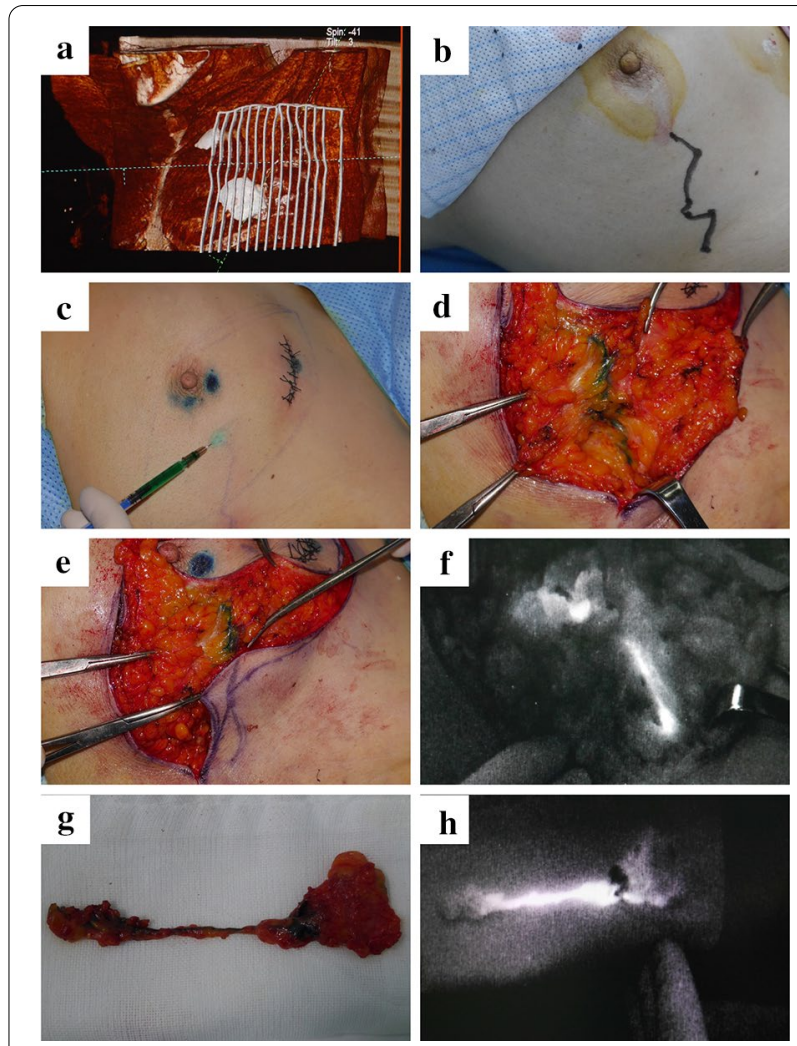

Fig. 2 A typical case to illustrate the consistency between preoperative localization and intraoperative detection. a The image of three-dimensional reconstruction based on CTLG. b The preoperative localization of SLN and LV on body surface according to CTLG. C Single point injection of methylene blue and indocyanine green. d-e Comparison between preoperative localized and intraoperative blue-stained LV. $\mathbf{f}$ Comparison between preoperative localized and intraoperative fluorescent LV. $\mathbf{g}-\mathbf{h}$ Comparison between blue-stained and fluorescent LV and SLN

and indocyanine green fluorescence during operation. In total, 40 SLNs were detected using preoperative CTLG, and 42 SLNs were detected using intraoperative combined stain. The accuracy rate of SLN number detection using preoperative CTLG was $95.2 \%$ (40/42). A total of 35 lymphatics were detected through preoperative CTLG; however, more lymphatic trunks were detected during the operation in the 2 patients.

\section{Selection of operative incision and single point injection of stains based on preoperative CTLG}

To protect the lymphatics, an operative incision was made far from the preoperatively localized lymphatic drainage pathways (Fig. 3a-b). After tumor excision, methylene blue and indocyanine green were subcutaneously injected to a single point, namely, the initial point of lymphatics that was mapped preoperatively (Fig. 3c). In the following operation, the course of blue-stained 

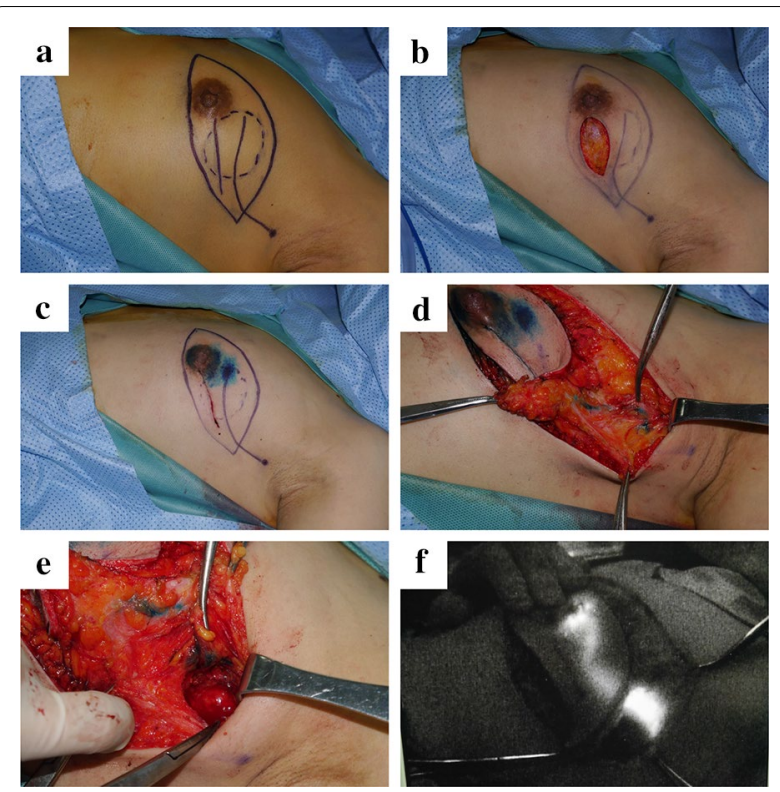

Fig. 3 The intraoperative application of preoperative localization of SLNs and lymphatics. a Determine the operative incision according to preoperative localization. $\mathbf{b}$ Excise the mass far from the preoperatively localized lymphatic drainage pathways. c Single point injection of methylene blue and indocyanine green. $\mathbf{d}$ Explore the course of blue-stained lymphatic. e Localized the blue-stained SLN. f Detection the fluorescence after indocyanine green staining

lymphatics was carefully explored and recorded (Fig. 3d). Therefore, SLNs were found to be localized at the end of the lymphatics (Fig. 3e). Then, a near-infrared camera was used to further confirm the identity of the SLNs by detecting the fluorescence of the lymph nodes (Fig. 3f).

\section{Discussion}

SLNB has gradually replaced ALND as the standard treatment for early-stage breast cancer patients without lymph node metastasis. SLNs are commonly identified by the combination of blue dye and radioactive tracer; however, this gold standard method is not feasible in many medical institutions, and the combination of indocyanine green and blue dye was introduced with a high identification rate for SLNs. Moreover, the reliability and safety of the SLNB procedure is dependent on the experience of surgeons [31], and even SLNB-mastered surgeons cannot avoid the identification failure of SLNs. In addition, some patients might prefer excision biopsy rather than FNAC or CNB due to the higher risk of forming hematomas or infections, cancer spreading, or the existence of false negative rate, which might cut off the LVs of the SLNs, leading to inability of dye and tracer to reach the SLNs and ultimate detection failure of SLNs intraoperatively. Therefore, preoperative localization of the lymphatic drainage pathway is helpful to improve the accuracy of SLNB.

In this study, we revealed the accurate localization of SLNs and LVs based on CTLG on the body surface of breast cancer patients, and the identification rate of SLNs was $100 \%$, which was higher than that noted in previous studies $(67-98 \%)$ [32, 33]. Moreover, the mean number of SLNs detected by CTLG was reported to be 1.1-3.1 [34], and our result was 1.5. CTLG could also help to completely understand the anatomical structure of the lymphatic pathway and the connection pattern between lymph nodes and lymph vessels, which has great potential in the study of the breast lymphatic system. Previous studies revealed that the most commonly observed LVSLN connection pattern is one LV connecting with one SLN [33], which is consistent with our finding. In addition, CTLG information may help to predict axillary lymph node metastasis, as CTLG clearly shows enlarged lymph nodes. Although the breast was slightly enlarged due to retention of contrast agent in the connective tissue space, no other discomfort or complication occurred, and it could rehabilitate within $2-3 \mathrm{~h}$ after injection. Therefore, these results indicated that CTLG had satisfactory accuracy, feasibility, and safety in preoperative SLN localization.

Preoperative SLN and lymphatic localization was consistent with intraoperative detection in 25/27 patients, and all SLNs and lymphatics visualized by CTLG were detected during the operation, indicating a high accuracy of CTLG. Importantly, CTLG could also accurately locate SLNs and their lymphatics even in patients with multiple SLNs and more complex lymphatic drainage pathways. Most tumors were reported to be located in the upper outer quadrant (UOQ) of the breast [35]. As shown in our study, most of the lymphatics flowing to SLNs were also located in the UOQ of the breast. Therefore, there is a potential possibility of cutting off lymphatics when the tumor is excised, which inevitably leads to failure of SLN detection. Among the 27 patients, 3 patients had a lymphatic drainage pathway passing through or across the tumor. To avoid cutting off the lymphatic vessels, the operative incision was far from the lymphatic vessels according to the preoperative localization via CTLG. As a result, all 3 patients had complete blue-stained lymphatic vessels and fluorescent sentinel lymph nodes. Preoperative localization of SLNs could facilitate the detection of SLNs and therefore speed up the SLNB procedure [33]. Moreover, the preoperative localization of SLNs can help to reduce the anxiety of surgeons and make the identification rate less dependent on the experience of surgeons. Furthermore, there is discussion about the injection site of tracers. In some centers, periareolar injection is preferred [36], while four quadrant injections have also 
been reported in several studies [36]. However, satisfactory detection rates of SLNs have been reported for all injection approaches [37]. In this study, we injected the tracer at a single initial point of the lymphatic drainage pathway, which was mapped via CTLG rather than multipoint injection based on the experience of surgeons. As a result, the SLNs were all successfully detected, no complications (such as skin tattoos, pain at injection sites, local skin reactions) were observed.

Accurate distinction between SLNs and non-SLNs is significant for SLNB given that removal of non-SLNs could increase the incidence of postoperative complications, including lymphedema $[38,39]$, potentially due to unnecessary removal of arm reversing lymph nodes and disrupted lymphatic drainage. In this study, we found that the second-tier nodes after the SLNs were also enhanced due to the overflow of iopamidol, which may be identified as SLNs in the operation. Moreover, some LNs around the SLNs, which were not enhanced using CTLG, may also be stain blue due to the spillover effect of blue dyes, making it difficult to distinguish them from the SLNs. The exact number of SLNs that should be removed in SLNB is also controversial [40-42]. A previous study reported that a negative correlation exists between the dissected number of SLNs and false-negative rates [43], which made surgeons remove more non-SLNs to attain lower false-negative rates. One way to solve this problem is to clarify the drainage relationship between SLNs and their surrounding LNs. Our previous study reported that true SLNs were defined as LNs that first received lymphatic drainage, which could be identified with the help of precise localization of the LVs and LNs [44]. In this study, we found that CTLG could provide a good depiction of lymphatic drainage pathways. Although we found that some LNs after the SLNs were also enhanced due to the overflow of iopamidol, they were not defined as true-SLNs. Therefore, our study presents a feasible method that enables the precise identification of trueSLNs guided by lymphatic drainage pathway, contributing to accurately localizing SLNs and distinguishing true SLNs from non-SLNs, including arm reversing nodes, in breast cancer.

The distribution of iopamidol in the lymphatic system may depend on the injection volume, fluidity, and the number, size, and integrity of the associated LVs and LNs [45]. In the study, the LVs were distinctly enhanced in patients with strongly enhanced LNs, and patients with weaker LN signals also had weaker LV signals, indicating that more iopamidol would enter the LVs and LNs of individuals with higher openness of the lymphatic system. For incomplete lymphography, 3/5 cases showed absent visualization of the initial segment, $1 / 5$ cases had an interruption in the middle segment, and 1/5 cases had no visualization of the terminal segment. The quickly passing iopamidol may lead to the absence of the initial segment, and the possible explanation for the absence of the middle and terminal segments is that a piston effect exists in lymphatic vessels [46]. Moreover, our study revealed that the time interval before lymphatic visualization was different, which may be related to the slow transportation and isolation of iopamidol in lymph node sinusoids [45]. Gentle massage of the injection site can promote the flow of iopamidol and its accumulation in SLNs [47], which is beneficial to shorten the examination time.

However, there are some limitations in our study. The number of patients is relatively small. There is no information about the adjuvant treatment and prognosis of those patients, so the prognostic value of this method cannot be quantified. Therefore, more studies are needed to evaluate the clinical value of CTLG.

\section{Conclusion}

Our results revealed that CTLG is a feasible method for the preoperative localization of SLNs in breast cancer patients with nearly no exposure to radiation, no need for facility approval, and low cost, which could help to select the precise injection site and time of massage during SLNB and improve the identification rate of SLNs. Moreover, SLNB could be performed well for a doctor with little experience in SLNB with the help of CTLG. The use of CTLG would be a valuable complimentary tool for identifying SLNs in institutions that cannot use the radioisotope method.

\section{Abbreviations}

SLNB: Sentinel lymph node biopsy; CTLG: Computed tomographic lymphography; ALND: Axillary lymph node dissection.

\section{Supplementary Information}

The online version contains supplementary material available at https://doi. org/10.1186/s12893-021-01190-7.

Additional file 1: Table S1. Characteristics of patients. Table S2. Category standard of CTLG. Table S3. Pattern of LV-SLN connection. Table S4. Initial point of lymphatic drainage pathway.

\section{Acknowledgments}

Not applicable.

\section{Authors' contributions}

SW: conceptualization, visualization, methodology, writing original draft. YL: validation, data curation, investigation, writing-original draft. XK: data curation, investigation, resources. BL: methodology, investigation, resources. TM: investigation, resources. YZ: investigation, resources. LJ: investigation, resources. $\mathrm{XL}$ : software, formal analysis. QY: writing-review \& editing, conceptualization, project administration, supervision. All authors read and approved the final manuscript. 


\section{Funding}

This work was supported by the National Natural Science Foundation of China (No. 81672613; No. 81874119; No. 82072912), Special Foundation for Taishan Scholars (No. ts20190971), Special Support Plan for National High-Level Talents (Ten Thousand Talents Program W01020103), National Key Research and Development Program (No. 2018YFC0114705), Clinical Research Center of Shandong University (No.2020SDUCRCA015), Qilu Hospital Clinical New Technology Developing Foundation (No. 2018-7; No. 2019-3).

\section{Availability of data and materials}

The datasets used and analyzed during the current study are available from the corresponding author on reasonable request.

\section{Declarations}

\section{Ethics approval and consent to participate}

All procedures of the present study were in accordance with the ethical standards of the institutional and national research committee and with the 1964 Helsinki Declaration and its later amendments or comparable ethical standards. The study was approved by the Ethical Committee of Qilu Hospital of Shandong University (KYLL-2016-231). The written informed consent was obtained from all patients.

\section{Consent for publication}

The written informed consent to publish the images and clinical details of the participants was obtained and copy of the written consent is available for review by the editor of this journal.

\section{Competing interests}

The authors declare that they have no competing interests.

\section{Author details \\ 1 Department of Breast Surgery, General Surgery, Qilu Hospital of Shandong University, Wenhua Xi Road No. 107, Jinan 250012, Shandong, China. ${ }^{2}$ Depart- ment of Head and Neck Surgery, Fudan University Shanghai Cancer Center, Shanghai, China. ${ }^{3}$ Department of Oncology, Shanghai Medical College, Fudan University, Shanghai, China. ${ }^{4}$ Department of Radiology, Qilu Hospital of Shandong University, Jinan, China. ${ }^{5}$ Pathology Tissue Bank, Qilu Hospital of Shandong University, Jinan, China. ${ }^{6}$ Research Institute of Breast Cancer, Shandong University, Jinan, China.}

Received: 7 December 2020 Accepted: 1 April 2021 Published online: 09 April 2021

\section{References}

1. Bray F, Ferlay J, Soerjomataram I, Siegel RL, Torre LA, Jemal A. Global cancer statistics 2018: GLOBOCAN estimates of incidence and mortality worldwide for 36 cancers in 185 countries. CA Cancer J Clin. 2018;68(6):394-424.

2. Liao GS, Chou YC, Hsu HM, Dai MS, Yu JC. The prognostic value of lymph node status among breast cancer subtypes. Am J Surg. 2015;209(4):717-24.

3. Ververs JM, Roumen RM, Vingerhoets AJ, Vreugdenhil G, Coebergh JW, Crommelin MA, Luiten EJ, Oj RVD, Schijven M, Wissing JC. Risk, severity and predictors of physical and psychological morbidity after axillary lymph node dissection for breast cancer. Eur J Cancer. 2001;37(8):991.

4. Kuehn T, Klauss W, Darsow M, Regele S, Flock F, Maiterth C, Dahlbender R, Wendt I, Kreienberg R. Long-term morbidity following axillary dissection in breast cancer patients_clinical assessment, significance for life quality and the impact of demographic, oncologic and therapeutic factors. Breast Cancer Res Treat. 2000;64(3):275-86.

5. Ashikaga T, Krag DN, Land SR, Julian TB, Anderson SJ, Brown AM, Ms JMS, Harlow SP, Weaver DL, Mamounas EP. Morbidity results from the NSABP B-32 trial comparing sentinel lymph node dissection versus axillary dissection. J Surg Oncol. 2010;102(2):111-8.

6. Purushotham AD, Upponi S, Klevesath MB, Bobrow L, Millar K, Myles JP, Duffy SW. Morbidity after sentinel lymph node biopsy in primary breast cancer: results from a randomized controlled trial. J Clin Oncol. 2005;23(19):4312-21.

7. Veronesi U, Paganelli G, Galimberti V, Viale G, Zurrida S, Bedoni M, Costa A, De CC, Geraghty JG, Luini A. Sentinel-node biopsy to avoid axillary dissection in breast cancer with clinically negative lymph-nodes. Lancet. 1997;349(9069):1864

8. Tafra L. A randomized comparison of sentinel-node biopsy with routine axillary dissection in breast cancer. N Engl J Med. 2005;349(6):546-53.

9. Lyman GH, Giuliano AE, Somerfield MR, Benson AB, Bodurka DC, Burstein HJ, Cochran AJ, Cody HS, Edge SB, Galper S. American society of clinical oncology guideline recommendations for sentinel lymph node biopsy in early-stage breast cancer. J Clin Oncol. 2005;23(30):7703.

10. Giuliano AE, Hunt KK, Ballman KV, Beitsch PD, Whitworth PW, Blumencranz PW, Leitch AM, Saha S, Mccall LM, Morrow M. Axillary dissection vs no axillary dissection in women with invasive breast cancer and sentinel node metastasis: a randomized clinical trial. JAMA. 2011;305(6):569.

11. Giuliano AE, McCall L, Beitsch P, Whitworth PW, Blumencranz P, Leitch AM, Saha S, Hunt KK, Morrow M, Ballman K. Locoregional recurrence after sentinel lymph node dissection with or without axillary dissection in patients with sentinel lymph node metastases: the American College of Surgeons Oncology Group Z0011 randomized trial. Ann Surg. 2010;252(3):426-32 (discussion 432-423)

12. Lyman GH, Somerfield MR, Bosserman LD, Perkins CL, Weaver DL, Giuliano AE. Sentinel lymph node biopsy for patients with early-stage breast cancer: American Society of Clinical Oncology clinical practice guideline update. J Clin Oncol. 2017;35(5):561-4.

13. Qin X, Yang M, Zheng X. Comparative study of indocyanine green combined with blue dye with methylene blue only and carbon nanoparticles only for sentinel lymph node biopsy in breast cancer. Ann Surg Treat Res. 2019;97(1):1-6

14. Senkus E, Kyriakides S, Ohno S, Penault-Llorca F, Poortmans P, Rutgers E, Zackrisson S, Cardoso F, Committee EG. Primary breast cancer: ESMO Clinical Practice Guidelines for diagnosis, treatment and follow-up. Ann Oncol. 2015;26(Suppl 5):v8-30.

15. Uematsu T, Kasami M. The use of positive core wash cytology to estimate potential risk of needle tract seeding of breast cancer: directional vacuum-assisted biopsy versus automated core needle biopsy. Breast Cancer. 2010;17(1):61-7.

16. Uematsu T, Kasami M. Risk of needle tract seeding of breast cancer: cytological results derived from core wash material. Breast Cancer Res Treat. 2008;110(1):51-5

17. Fitzal F, Sporn EP, Draxler W, Mittlbock M, Taucher S, Rudas M, Riedl O, Helbich TH, Jakesz R, Gnant M. Preoperative core needle biopsy does not increase local recurrence rate in breast cancer patients. Breast Cancer Res Treat. 2006;97(1):9-15.

18. Hoorntje LE, Schipper ME, Kaya A, Verkooijen HM, Klinkenbijl JG, Borel Rinkes IH. Tumour cell displacement after 14G breast biopsy. Eur J Surg Oncol. 2004;30(5):520-5.

19. Calvillo KZ, Guo L, Brostrom V, Schnitt SJ, Hong X, Raza S, Lester SC. Recurrence of breast carcinoma as Paget disease of the skin at a prior core needle biopsy site: case report and review of the literature. Int J Surg Case Rep. 2015;15:152-6.

20. Liikanen J, Leidenius M, Joensuu H, Vironen J, Heikkila P, Meretoja T. Breast cancer prognosis and isolated tumor cell findings in axillary lymph nodes after core needle biopsy and fine needle aspiration cytology: biopsy method and breast cancer outcome. Eur J Surg Oncol. 2016;42(1):64-70.

21. Chen AM, Haffty BG, Lee CH. Local recurrence of breast cancer after breast conservation therapy in patients examined by means of stereotactic core-needle biopsy. Radiology. 2002;225(3):707-12.

22. Hao S, Liu ZB, Ling H, Chen JJ, Shen JP, Yang WT, Shao ZM. Changing attitudes toward needle biopsies of breast cancer in Shanghai: experience and current status over the past 8 years. Onco Targets Ther. 2015;8:2865-71.

23. Mitra S, Dey P. Fine-needle aspiration and core biopsy in the diagnosis of breast lesions: a comparison and review of the literature. Cytojournal. 2016;13:18.

24. Tham TM, lyengar KR, Taib NA, Yip CH. Fine needle aspiration biopsy, core needle biopsy or excision biopsy to diagnose breast cancer-which is the ideal method? Asian Pac J Cancer Prev. 2009;10(1):155-8.

25. Tan H, Yang B, Wu J, Wana S, Gu Y, Li W, Jiang Z, Qian M, Peng W. Localization and evaluation of sentinel lymph node in breast cancer 
from computed tomographic lymphography. J Comput Assist Tomogr. 2011;35(3):367-74.

26. Kim K, Cheon S, Kang K, Hwang Y, Oh D, Yoon J, Choi M. Computed tomographic lymphangiography of the thoracic duct by subcutaneous iohexol injection into the metatarsal region. Vet Surg. 2020;49(1):180-6.

27. Honda K, Ishiyama K, Suzuki S, Oumi E, Sato T, Kawasaki Y, Saito H, Ishikawa K. Sentinel lymph node biopsy using computed tomographic lymphography in patients with early tongue cancer. Acta Otolaryngol. 2015;135(5):507-12.

28. Minohata J, Takao S, Hirokaga K. Sentinel lymph node biopsy using CT lymphography in breast cancer. Breast Cancer. 2011;18(2):129-36.

29. Yuasa Y, Seike J, Yoshida T, Takechi H, Yamai H, Yamamoto Y, Furukita Y, Goto M, Minato T, Nishino T, et al. Sentinel lymph node biopsy using intraoperative indocyanine green fluorescence imaging navigated with preoperative CT lymphography for superficial esophageal cancer. Ann Surg Oncol. 2012;19(2):486-93.

30. Yang B, Tan H, Chen J, Wang S, Li W, Peng W, Han Q, Shen Z, Shao Z, Wu J. A novel technique for localization of sentinel lymph node in breast cancer patients based on computed tomographic lymphography. Breast J. 2011;17(4):431-3.

31. Mcmasters KM, Wong SL, Chao C, Woo C, Tuttle TM, Noyes RD, Carlson DJ, Laidley AL, Mcglothin TQ, Ley PB. Defining the optimal surgeon experience for breast cancer sentinel lymph node biopsy: a model for implementation of new surgical techniques. Ann Surg. 2001;234(3):292-9.

32. Ashiba H, Nakayama R. Computerized evaluation scheme to detect metastasis in sentinel lymph nodes using contrast-enhanced computed tomography before breast cancer surgery. Radiol Phys Technol. 2019;12(1):55-60.

33. Yokohata K, Hattori M, Fujimoto T, Chikazawa N, Maeyama R, Yamanaka N, Kuroki H, Sasaki N, Kameoka N, Matoba N. Computed tomographic lymphography predicts the difficulty of sentinel lymph node biopsy with dye. Breast Cancer. 2014;21(2):198-201.

34. Motomura K, Sumino H, Noguchi A, Horinouchi T, Nakanishi K. Sentinel nodes identified by computed tomography-lymphography accurately stage the axilla in patients with breast cancer. BMC Med Imaging. 2013;13:42

35. Darbre PD. Recorded quadrant incidence of female breast cancer in Great Britain suggests a disproportionate increase in the upper outer quadrant of the breast. Anticancer Res. 2005;25(3c):2543.

36. Shimazu K, Tamaki Y, Taguchi T, Takamura Y, Noguchi S. Comparison between periareolar and peritumoral injection of radiotracer for sentinel lymph node biopsy in patients with breast cancer. Surgery. 2002;131(3):277-86
37. Giammarile F, Alazraki N, Aarsvold JN, Audisio RA, Glass E, Grant SF, Kunikowska J, Leidenius M, Moncayo VM, Uren RF, et al. The EANM and SNMMI practice guideline for lymphoscintigraphy and sentinel node localization in breast cancer. Eur J Nucl Med Mol Imaging. 2013:40(12):1932-47.

38. Wilke LG, McCall LM, Posther KE, Whitworth PW, Reintgen DS, Leitch AM, Gabram SG, Lucci A, Cox CE, Hunt KK, et al. Surgical complications associated with sentinel lymph node biopsy: results from a prospective international cooperative group trial. Ann Surg Oncol. 2006;13(4):491-500.

39. Boneti C, Korourian S, Bland K, Cox K, Adkins LL, Henry-Tillman RS, Klimberg VS. Axillary reverse mapping: mapping and preserving arm lymphatics may be important in preventing lymphedema during sentinel lymph node biopsy. J Am Coll Surg. 2008;206(5):1038-42.

40. Zakaria S, Degnim AC, Kleer CG, Diehl KA, Cimmino VM, Chang AE, Newman LA, Sabel MS. Sentinel lymph node biopsy for breast cancer: how many nodes are enough? J Surg Oncol. 2010;96(7):554-9.

41. Ban EJ, Lee JS, Koo JS, Park S, Kim SI, Park BW. How many sentinel lymph nodes are enough for accurate axillary staging in $\mathrm{T1}-2$ breast cancer? J Breast Cancer. 2011;14(4):296-300.

42. Lynch MA, Jackson J, Kim JA, Leeming RA. Optimal number of radioactive sentinel lymph nodes to remove for accurate axillary staging of breast cancer. Surgery. 2008;144(4):525-32.

43. KennedyKollias RJJ, Gill PG, Bochner M, Coventry BJ, Farshid G. Removal of two sentinel nodes accurately stages the axilla in breast cancer. Br I Surg. 2010;90(11):1349-53.

44. Li X, Chen S, Jiang L, Kong X, Ma T, Xu H, Yang Q. Precise intraoperative sentinel lymph node biopsies guided by lymphatic drainage in breast cancer. Oncotarget. 2017;8(38):63064-72.

45. Ruehm SG, Corot C, Debatin JF. Interstitial MR lymphography with a conventional extracellular gadolinium-based agent: assessment in rabbits. Radiology. 2001;218(3):664-9.

46. Tangoku A, Yamamoto S, Suga K, Ueda K, Nagashima Y, Hida M, Sato T, Sakamoto K, Oka M. Sentinel lymph node biopsy using computed tomography-lymphography in patients with breast cancer. Surgery. 2004;135(3):258-65.

47. Mariani G, Moresco L, Viale G, Villa G, Bagnasco M, Canavese G, Buscombe J, Strauss HW, Paganelli G. Radioguided sentinel lymph node biopsy in breast cancer surgery. J Nucl Med. 2001;42(8):1198-215.

\section{Publisher's Note}

Springer Nature remains neutral with regard to jurisdictional claims in published maps and institutional affiliations.
Ready to submit your research? Choose BMC and benefit from:

- fast, convenient online submission

- thorough peer review by experienced researchers in your field

- rapid publication on acceptance

- support for research data, including large and complex data types

- gold Open Access which fosters wider collaboration and increased citations

- maximum visibility for your research: over $100 \mathrm{M}$ website views per year

At BMC, research is always in progress.

Learn more biomedcentral.com/submissions 